\section{Successful triple immuno- enzymatic method employing primary antibodies from same species and same immunoglobulin subclass}

T.A. Osman, ${ }^{1,2}$ G. Oijordsbakken, ${ }^{1}$

D.E. Costea, ${ }^{1}$ A.C. Johannessen ${ }^{1,3}$

'Department of Clinical Medicine, The Gade Laboratory for Pathology, Faculty of Medicine and Dentistry, University of Bergen

2Department of Global Public Health

and Primary Care, Center for

International Health, Faculty of Medicine

and Dentistry, University of Bergen

${ }^{3}$ Department of Pathology, Haukeland

University Hospital, Bergen, Norway

\section{Abstract}

Protocols for immunohistochemical (IHC) detection of multiple antigens in the same tissue sections have been developed using primary antibodies directly conjugated to different enzymes or fluorochromes, or ones that have been raised in different species, or from different immunoglobulin (Ig) classes or subclasses. For antibodies lacking such dissimilarities, very few proposals have been published with varying degrees of generalizability. In this report we present a successful triple IHC protocol engaging three unconjugated monoclonal primary antibodies raised in the same species and of the same Ig subclass. Compared to other methods, our results showed that denaturation of the preceding reaction complex by microwave heating, combined with additional suppression of enzyme activity, enabled the detection of all three reactions by using the same detection system, with no cross reaction observed. Moreover, expression patterns of each of the three antigens in the triple stained sections, was found to be similar to the pattern observed when single staining was performed. Unlike previous reports, no damage of targeted antigens or tissues did occur following this protocol. Furthermore, the contrast of the colors employed was investigated by computerized color deconvolution, and the three reactions products were successfully separated into three individual images that could be used for further objective quantification.

\section{Introduction}

Detection of multiple tissue antigens can be achieved either by performing immunohistochemistry (IHC) for the targeted antigens individually in serial sections, or by applying multiple IHC in a single tissue section. In addition to being technically challenging to obtain serial sections as close as possible to each other, the topographical and co-localization information are affected by the thickness of the tissue section relative to the size of the cell type under investigation. ${ }^{1}$ In contrary, multiple IHC in a single tissue section would preserve the sample in cases where the size of sample is critical, and yet provides more informative results. Although this report will only focus on immunoenzyme method and the term IHC will be used accordingly, it is noteworthy that immunofluorescence (IF) is often preferred when targeted antigens are expected to colocalize at the same subcellular compartment. Nevertheless, the immunoenzyme method provides unwavering staining and fine morphological details that can be reviewed under a regular light microscope.

In multiple IHC, unwanted cross-reactivity between primary and secondary antibodies from different staining rounds is regarded as the main impediment, and many approaches have been suggested to circumvent it. The earliest proposed approach was the use of enzyme conjugated antibodies, albeit the limited applicability of direct IHC. ${ }^{2}$ On the other hand, the generally more applicable indirect IHC inherits higher risk of cross-reactivity unless the secondary antibodies to be used can specifically target individual primary antibody. ${ }^{1}$ Specificity of secondary antibodies is generally based on the species used to raise primary antibody and immunoglobulin (Ig) class or subclass. Nowadays, most of the commercially available antibodies are raised in mouse, rat or rabbit as IgGl, due to the relative ease of animal maintenance and other technical challenges. Therefore, it is a frequently encountered problem that required antibodies are available only from same species and Ig subclass. For such situations, the use of monovalent $\mathrm{F}\left(\mathrm{ab}^{1}\right)$ secondary antibody fragments has been proposed. ${ }^{3}$ Having only one antigen binding site, would lower their probability to cross-bind to subsequent primary antibodies. However, the relative low affinity of $F\left(a b^{1}\right)$ antibodies demands using high concentration to achieve detectable results, which often results in an increased noise to signal ratio. ${ }^{4}$ Another approach is to make use of the observation that 3,3 -diaminobenzidine tetrahydrochloride (DAB), a commonly used chromogen, can deposit around the antigen-antibody complex prohibiting them from cross-binding with sub-
Correspondence: Tarig A. Osman, The Gade Laboratory for Pathology, University of Bergen, Haukeland University Hospital, Central block, 2nd floor, N-5021 Bergen, Norway.

E-mail: tos094@gades.uib.no

Key words: triple immunohistochemistry, immunoenzyme, same species antibodies.

Acknowledgments: Special thanks for the Norwegian Dental Depots Fund (2011 and 2012), Gades legat (Dr.Med. F. G. Gades Legat, 2012), Norwegian Cancer Research Association and Bergen Medical Research Foundation (20/2009), for financial support.

The authors would also like to express their gratitude to Kathrine Skarstein for evaluation of the lymph nodes slides, Edith Fick for technical assistance, Sabine Leh and Charles Best for their assistance with the image analyses.

Contributions: TA0, GØ, immunohistochemistry experiments designing and performing, evaluation of sections, manuscript writing and reviewing; DEC, ACJ, study general design, immunohistochemistry experiments design, evaluation of sections, manuscript writing and reviewing.

Conflict of interests: none to declare.

Received for publication: 16 March 2013.

Accepted for publication: 21 June 2013.

This work is licensed under a Creative Commons Attribution NonCommercial 3.0 License (CC BYNC 3.0).

(OCopyright T.A. Osman et al., 2013

Licensee PAGEPress, Italy

European Journal of Histochemistry 2013; 57:e22 doi:10.4081/ejh.2013.e22

sequent antibodies. ${ }^{5}$ This observation is referred to as sheltering effect of DAB. Nevertheless, it is influenced by the concentration of the primary antibody, and it can have undesired influence on co-localizing antigens. ${ }^{6}$

Apart from the previously mentioned protocols, all other proposed approaches rely on targeting the preceding reaction either by saturation (blocking), stripping, or denaturation steps, prior to incubation with subsequent primary antibody from same species and Ig subclass. Saturation of preceding reaction complex with an unbinding Ig from a species equivalent to primary antibodies, followed by a secondary monovalent $\mathrm{F}\left(\mathrm{ab}^{1}\right)$ fragment against it, has been reported. ${ }^{7}$ Despite its elegance, this approach is time consuming, and others failed to reproduce it. ${ }^{8}$ Moreover, a blocking step is also a part of the EnVision ${ }^{\mathrm{TM}} G \mid 2$ Doublestain System from Dako (Dako, Golstrup, Denmark), although the mechanism is publicly unavailable. On the other hand, 
many methods for stripping of tissue bound antibodies have been published. Although destruction of some of the tissue antigens and removal of some chromogens is expected, the use of acidic or oxidative solutions has been reported. ${ }^{9-11}$ In 1995 , Lan et al. ${ }^{12}$ reported that two rounds of 5 min heating in a microwave oven denatured antigen-antibody complex from the preceding reaction, and enabled the subsequent detection of another primary antibody from same species, using the same secondary and tertiary immunoreagents. However, the protocol had certain limitations regarding detection of antigens at the cell membrane, or enzymes in the cytoplasm, particularly in formalin fixed paraffin embedded samples. Optimum time and microwave power to be used for double IHC was recently published by Chan et al., ${ }^{13}$ and no destruction of tissue antigens was reported.

In the current study, we aimed to optimize a universal triple IHC protocol for using antibodies raised in same species and belonging to the same Ig subclass, on the basis of previously published methods, and for using objective computerized quantification. The use of microwave for denaturation of antibodies was weighed against sheltering effect of DAB, and the use of a commercially available blocking solution. Unlike previous publications using denaturation methods, in this study a cell surface receptor, an intracellular cytoplasmic enzyme and a nuclear marker were targeted. The chromogens used yielded a sufficient color contrast, evident by computerized color deconvolution, despite the close localization of the antigens.

\section{Materials and Methods}

\section{Samples preparation}

Formalin fixed paraffin embedded normal human oral mucosa samples (NHOM, n=30), cervical lymph nodes (CLN, $\mathrm{n}=5$ ) and human testicular tissue (HT, $\mathrm{n}=1)$, were obtained from the archive of the Department of Pathology, Haukeland University Hospital,

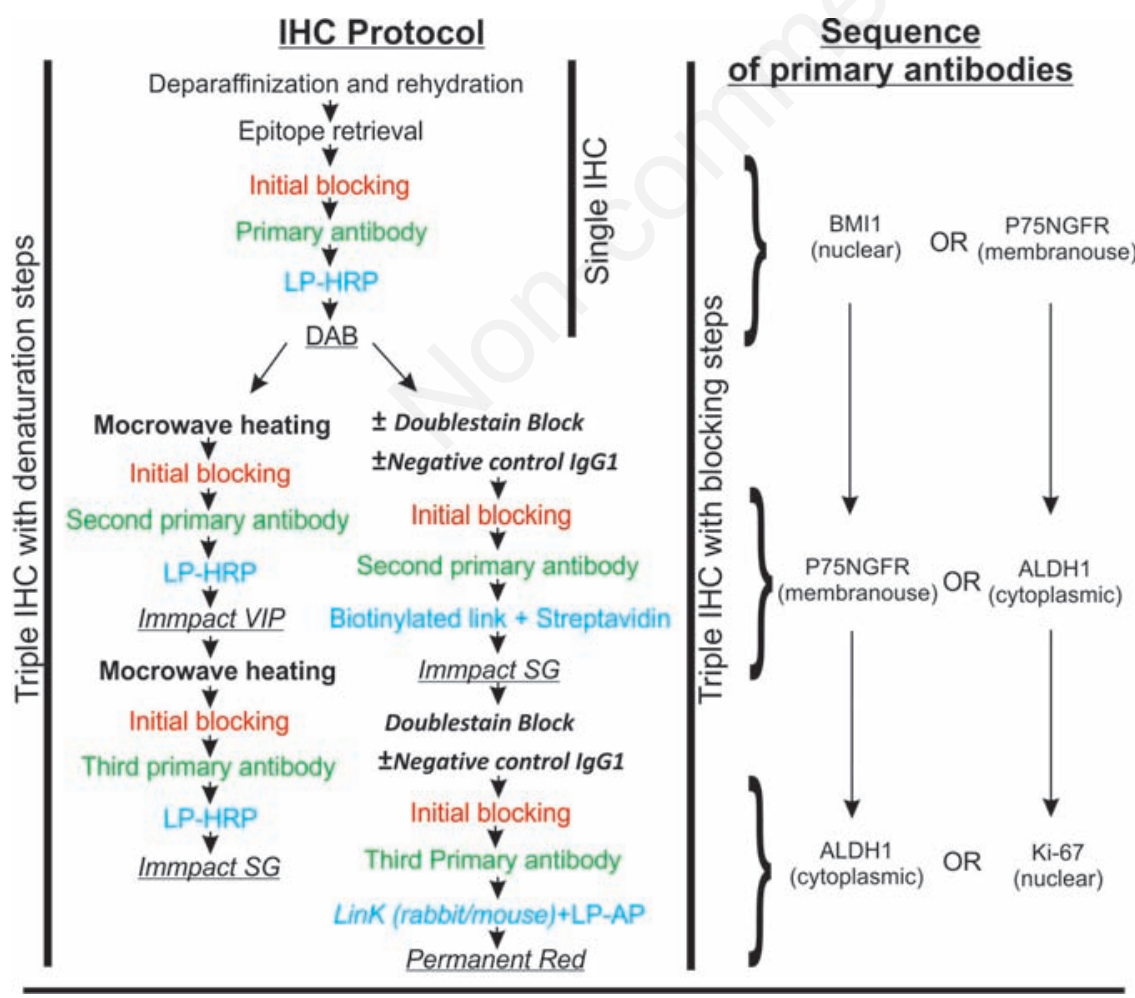

Figure 1. Flow chart illustrating the immunohistochemistry protocol, and sequence of primary antibodies. Chromogens used are underlined.

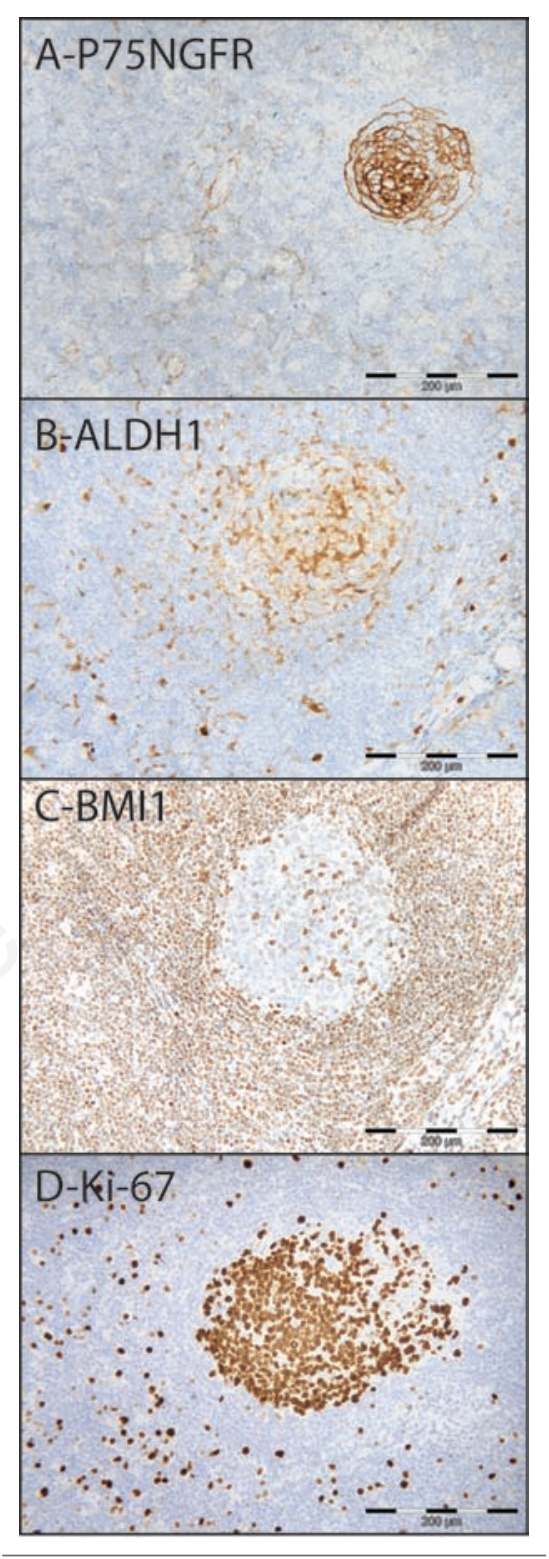

Figure 2. Single IHC for P75NGFR (A), ALDH1 (B), BMI1 (C), Ki-67 (D), in a cervical lymph node.

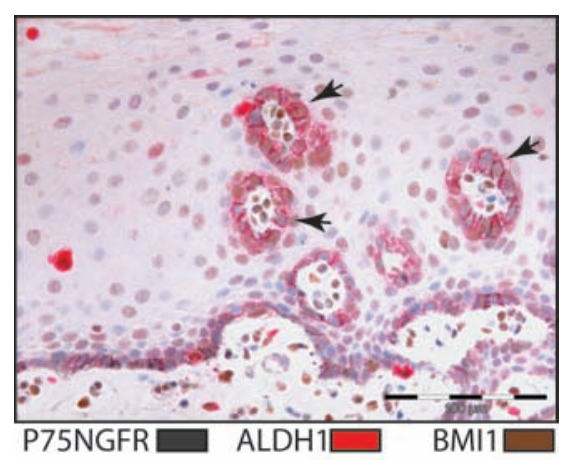

Figure 3. Triple staining for BMI1 (brown), ALDH1 (red) and P75NGFR (gray) in normal human oral mucosa. Doublestain block and Negative Control Mouse IgG1 were used for blocking. Arrows indicate sites of cross-reactions. 
Bergen, Norway. The study has been ethically approved by the Regional Committee for Medical Research Ethics in West Norway (REK vest, no. 2010/481-7).

Samples were cut into $4 \mu \mathrm{m}$ sections and kept at $56^{\circ} \mathrm{C}$ overnight. Afterwards, sections were deparaffinized by immersion in xylene for $18 \mathrm{~min}$, and rehydrated by graded alcohol concentrations to distilled water.

\section{Epitope retrieval}

Decloaking Chamber ${ }^{\mathrm{TM}}$ Plus (Biocare Medical, California, USA) was used for epitope retrieval. Sections were submerged in a pH 6.0 Target Retrieval Buffer (Dako) and heated at $125^{\circ} \mathrm{C}$ for $35 \mathrm{~s}$. Sections were then left to cool down on the bench for $10 \mathrm{~min}$ and washed under running tap water for $5 \mathrm{~min}$.

\section{Initial blocking step}

Sections were incubated with either Peroxidase Block or Dual Endogenous Enzyme Block (Dako) for 10 min to quench endogenous peroxidase or alkaline phosphatase activity respectively. To block unspecific binding sites of secondary antibody, sections were incubated for $30 \mathrm{~min}$ in a $10 \%$ solution of either normal goat serum, or normal rabbit serum (Dako), diluted in 3\% bovine serum albumin (BSA) (Sigma-Aldrich, St. Louis, M0, USA) in Tris-buffered saline (TBS).

\section{Primary antibodies}

The following monoclonal mouse antibodies were used in the study: anti-BMI1 (Merk Millipore, MA, USA, 1:500 to 1:1500), targeting the transcription factor polycomb complex protein BMIl, located in the cell nucleus. AntiALDH1 (BD Biosciences, Franklin Lakes, NJ, USA, 1:250), targeting the enzyme aldehydede- hydrogenase-1, located in the cytoplasm with occasional nuclear location, anti-P75NGFR (Merk Millipore, 1:2500), targeting the lowaffinity nerve growth factor receptor located in the cell membrane, and anti-Ki67 (Dako, 1:500 to 1:1500), targeting mindbomb E3 ubiquitin protein ligase-1, located in the cell nucleus. Dilutions of all primary antibodies were determined by first adopting the recommendation of the manufacturers, and both antibody dilution and incubation time was adjusted until a sufficient signal to noise ratio was achieved. Eventually, incubation with all primary antibodies was performed overnight at $4^{\circ} \mathrm{C}$. Negative Control Mouse IgG1 directed against non-human antigens (Dako, 1:250) was used instead of the primary antibody for negative control. For multiple staining, primary antibodies were incubated sequentially. Anti-BMI1 and anti-Ki67 antibodies were not employed in the same experiment (Figure 1).

\section{Detection of the reaction in single immunohistochemistry}

Sections were incubated with labeled polymerhorseradish peroxidase conjugated to goat antimouse immunoglobulins (LP-HRP) (EnVision+ ${ }^{\circledR}$, Dako) for $30 \mathrm{~min}$ at room temperature. Reactions were visualized using DAB (Dako).

\section{Detection of the reaction in triple immunohistochemistry}

To investigate the sheltering effect of DAB, the first reaction was developed following the procedure for single IHC. Subsequent reactions followed as illustrated in Figure 1. In order to avoid cross-reactivity with subsequent staining rounds, an intermediate step of either denaturation or blocking was performed as described below.

\section{Blocking step}

Blocking of preceding reaction complex was done before incubation with subsequent primary antibody. To achieve this, incubation with a ready-to-use blocking solution (Doublestain Block, Dako) for 3 min was performed. In some sections this was preceded by $1 \mathrm{~h}$ incubation with Negative Control Mouse IgG1 (1:250) at room temperature. Subsequent reactions were detected using different immunoreagents and chromogens respectively as follows: i) Mixture of biotinylated goat antimouse and anti-rabbit immunoglobulins (Real Link $^{\circledR}$, Dako), followed by streptavidin conjugated to horseradish peroxidase (Real Streptavidin Peroxidase ${ }^{\circledR}$, Dako). This reaction was visualized with ImmPACT SG (Vectorlabs, Burlingame, CA, USA), resulting in a gray reaction product; ii) Dextran polymer coupled with secondary antibodies against mouse and rabbit immunoglobulins (Link rabbit/mouse ${ }^{\circledR}$, Dako), followed by labeled polymer-alkaline phosphatse anti-mouse/rabbit (LP-AP) (Dako), and the reaction was visualized using Permanent Red. Incubation with secondary immunoreagents was performed according to manufacturer`s instructions.

\section{Denaturation step}

The denaturation step followed the protocol proposed by Chan et al. ${ }^{13}$ with slight modifications. In short, sections were submerged in the same retrieval buffer as before in a plastic jar, and the slide rack was filled up with empty slides as dummies. In total three plastic jars containing racks full of slides were placed in a Y letter shape in the center of the microwave oven tray (NN-L564W, Panasonic, Osaka, Japan). The microwave oven was operated at 1000 Watts for $2 \mathrm{~min}$, then at 250 Watts for five and a half min. The jars with slides were then

Table 1. Summary of the results of single staining.

\begin{tabular}{llll} 
& Normal human oral mucosa & Cervical lymph node & Human testis \\
P75NGFR & Basal layer keratinocytes & Germinal and non-germinal center cells & Interstitial cells \\
BMI1 & $\begin{array}{l}\text { Fibroblasts, basal and suprabasal } \\
\text { layer keratinocytes }\end{array}$ & Germinal and non-germinal center cells & Cells at seminiferous tubules \\
\hline ALDH1 & $\begin{array}{l}\text { Fibroblasts, basal and suprabasal } \\
\text { layer keratinocytes }\end{array}$ & Germinal and non-germinal center cells & Cells at seminiferous tubules and intertistial cells \\
Ki-67 & Basal and suprabasal layer keratinocytes & Germinal and non-germinal center cells & Cells at seminiferous tubules \\
\hline
\end{tabular}


left to cool down on the bench for $10 \mathrm{~min}$. For some sections, before incubation with subsequent primary antibody, microwave heating was followed by another initial blocking step. All successive reactions were detected using LP-HRP. ImmPACT VIP or ImmPACT SG (Vectorlabs) was used to visualize subsequent reactions with a purple or a gray color, respectively. To measure the change in buffer temperature during the microwave cycle, the experiment was performed using dummy slides, and temperature of the buffer was measured at the start and end points, and at every two minutes during the cycle, using a mercury-in-glass laboratory thermometer.

\section{Washing}

Sections were submerged in Tris-Buffered Saline (Trizma ${ }^{\circledR}$ hydrochloride $0.42 \mathrm{mM} / \mathrm{L}$, Trizma base $2.36 \mathrm{mM} / \mathrm{L}$, pH 7.4) with $0.1 \%$ Tween, and put on the shaker for $5 \mathrm{~min}$. This was performed after every step, except after serum blocking.

\section{Dehydration and storage}

Eventually, sections were counterstained with hematoxylin (Dako) or not at all. All sections were immersed briefly in ascending alcohol concentration followed by xylene, and cover slipped using non-aqueous mounting medium (Histokitt, Glaswarenfabrik Karl Hecht, Sondheim/RhÖn, Germany).

\section{Evaluation of sections}

Sections were evaluated using Leica DM-LB light microscope, equipped with $63 \mathrm{X}$ objective.

\section{Color deconvolution}

To investigate the contrast of the colors resulted from the three successive reactions, Color Deconvolution Algorithm, version 9.1 (Aperio, CA, USA) was used to generate three individual intensity range markup images from a triple stained section, each of which showing the distribution of one of the three colors. This process was performed only on sections that showed no visible cross-reactivity under the light microscope. Sections that had been counterstained with hematoxylin were also excluded from the analysis. Slides were scanned using Scanscope ${ }^{\circledR}$ XT (Aperio) at 40X objective. The generated digital slides were viewed in ImageScope, version 12 (Aperio). Single stained structures within the triple stained sections served as a reference to obtain the red, green and blue (RGB) constituents for each of the targeted colors. Average RGB optical densities for each of the three colors were used as input parameters for the algorithm, and the three resulting images were generated accordingly.

\section{Results}

\section{Single himmunohistochemistry}

Primarily, single IHC were used to test the four antibodies individually. The patterns of expression of BMI1 and Ki-67 (nuclear), ALDH1 (cytoplasmic \pm nuclear) and P75NGFR (membranous) were found to be consistent with previous publications, ${ }^{14-21}$ or with the human atlas of proteins (Table 1, Figure 2) ${ }^{22}$ In comparison, sections incubated with negative control IgG1 or antibody diluent showed no positive reaction at all (data not shown).

\section{Multiple staining}

\section{Sheltering effect of chromogen}

Sheltering effect of DAB prohibited subsequent cytoplasmic immunoreactivity for antiALDH1 antibody when anti-BMI1 or anti-Ki67

Table 2. Summary of the co-localization of antigens in triple stained sections as compared to single staining.

\begin{tabular}{|c|c|c|c|c|c|c|c|c|c|}
\hline & \multicolumn{3}{|c|}{$\begin{array}{c}\text { Normal human } \\
\text { oral mucosa }\end{array}$} & \multicolumn{3}{|c|}{$\begin{array}{l}\text { Cervical } \\
\text { lymph node } \\
\text { P75 ALDH1 P75+ALDH1 }\end{array}$} & \multicolumn{3}{|c|}{ Human testis } \\
\hline BMI1 & + & + & + & + & + & + & + & + & - \\
\hline Ki67 & + & - & - & + & + & - & - & + & - \\
\hline
\end{tabular}

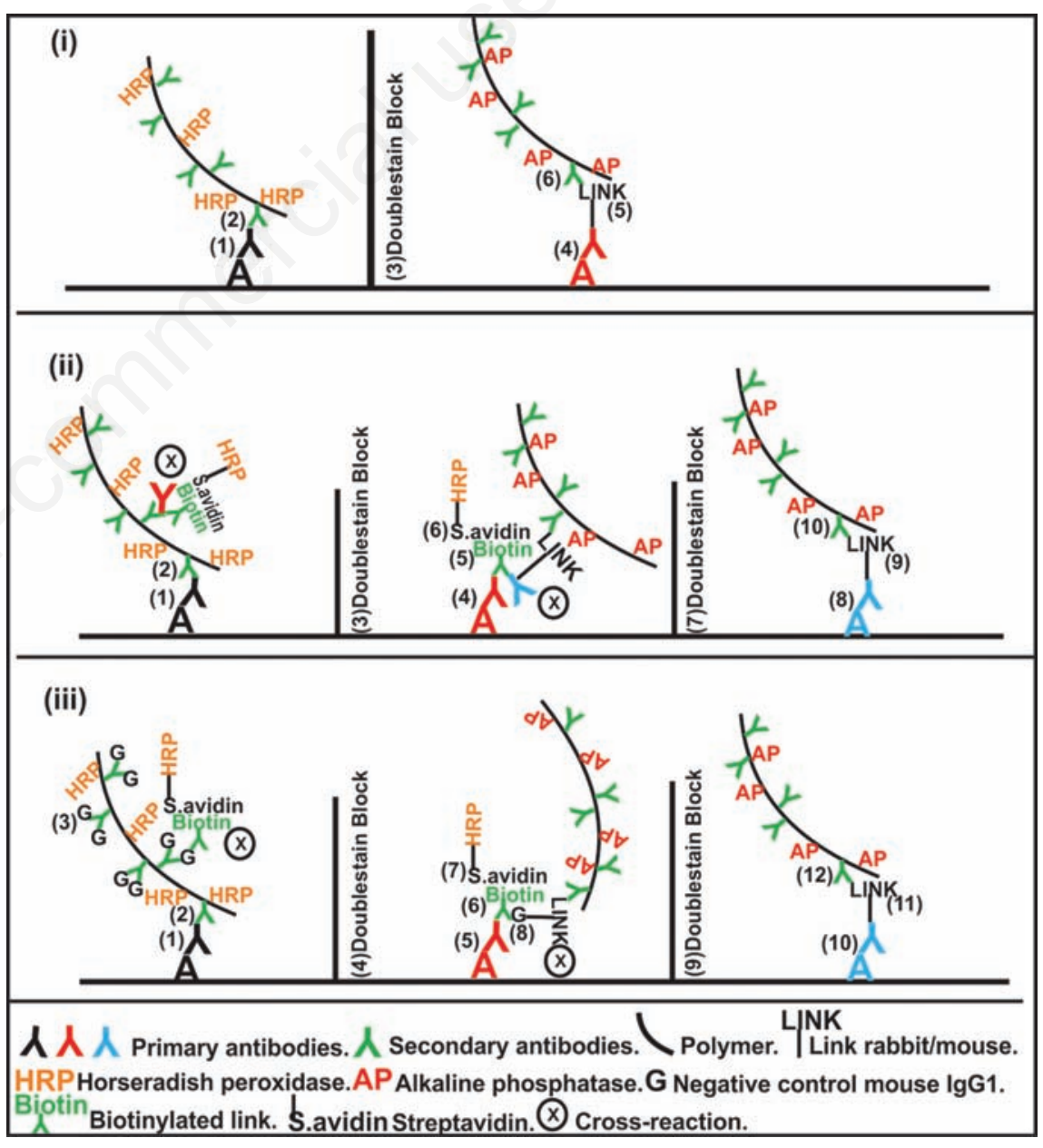

Figure 4. Schematic figure illustrating blocking of cross-reactions in multistaining procedures: recommended use of Doublestain Block (i), cross-reactions when using Doublestain Block (ii), cross-reactions when using Negative Control Mouse IgG1, in addition to Doublestain Block (iii). 
antibody was diluted as 1:500 (data not shown). High dilution (1:1500) of anti-BMI1 antibody, but not anti-Ki67 antibody eliminated this effect, and therefore Ki67 reaction was carried out at the third staining round.

Blocking

The use of Doublestain Block was not found to be effective in eliminating cross reactivity when used between reactions developed with LP-HRP, streptavidin-biotin method and LP-AP respectively (Figure 3 ). No difference was observed with additional use of Negative Control Mouse IgG1. Cross-reactivity was detected between successive reactions, but not between reactions detected with LP-HRP and LP-AP (Figure 4).

\section{Denaturation}

Buffer temperature readings are blotted against time in Figure 5. The use of microwave for denaturation of preceding reaction followed by an additional initial blocking step, enabled detection of all three reactions using LP-HRP. In this case, no cross reaction evident by spurious mixed color was detected, and the staining was found consistent with sections stained with individual primary antibodies (Table 2, Figures 6 and 7). Occasionally, the three reactions were detected in different subcellular compartments within the same cell. Incubation with Negative Control Mouse IgG1 instead of one of the primary antibodies resulted in a consistent double staining for the other two antibodies (Figure 8).

\section{Trouble shooting for triple staining}

i) Masking of nuclear immunoreactivity by cytoplasmic color: the color of DAB deposited by anti-ALDH1 antibody immunoreactivity in the cytoplasm, resulted in no visible nuclear immunoreactivity. This did not occur when ALDH1 reaction was visualized using other colors. ii) Vanishing of first reaction product: Using ImmPACT VIP or ImmPACT SG for visualization of first reaction showed a drastic effect of the microwave heating on the color intensity. Comparatively, this effect was less prominent when DAB was employed to visualize the first reaction. iii) Applying the method reported by Chan et al. ${ }^{13}$ in our setup, resulted in a mixed color on the cell membrane in some of the samples, which indicates a remaining enzyme activity at site of preceding reaction (data not shown). However, additional initial blocking step proved effective in eliminating the problem. iv) Weak hematoxylin color in sections subjected to the denaturation steps: doubling the time of incubation with hematoxylin resulted in sufficient counterstaining.

Applying the points mentioned in the trouble shooting part resulted in the sequence of reactions illustrated in Figure 1. Either a nuclear or a membranous reaction had been carried out first and visualized with DAB, and

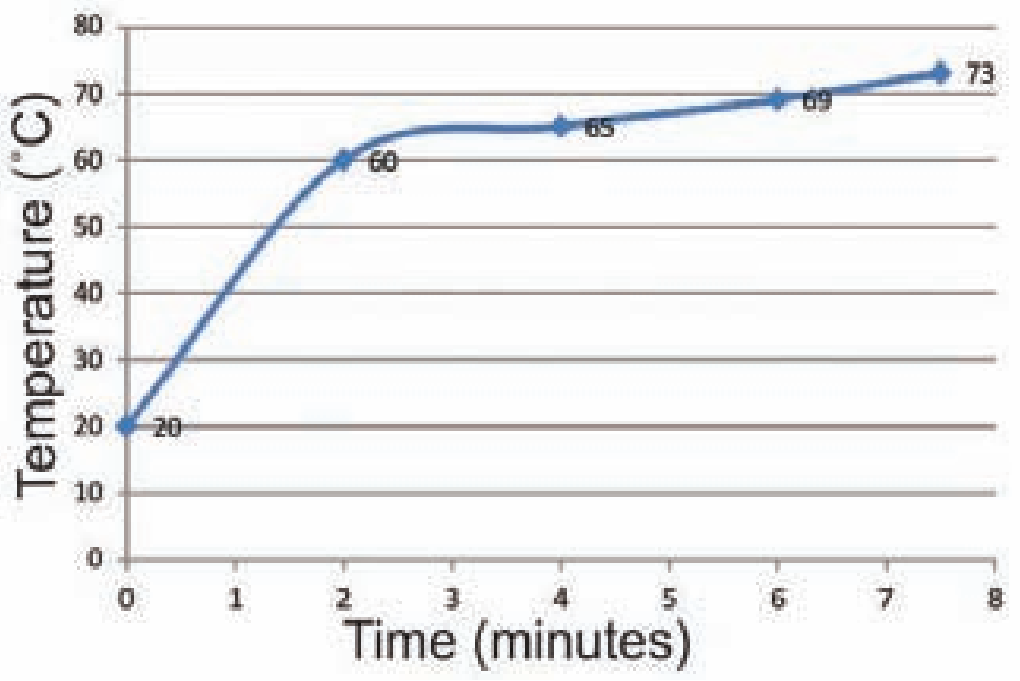

Figure 5. Line chart illustrating changes in buffer temperature over time, during the denaturation step.

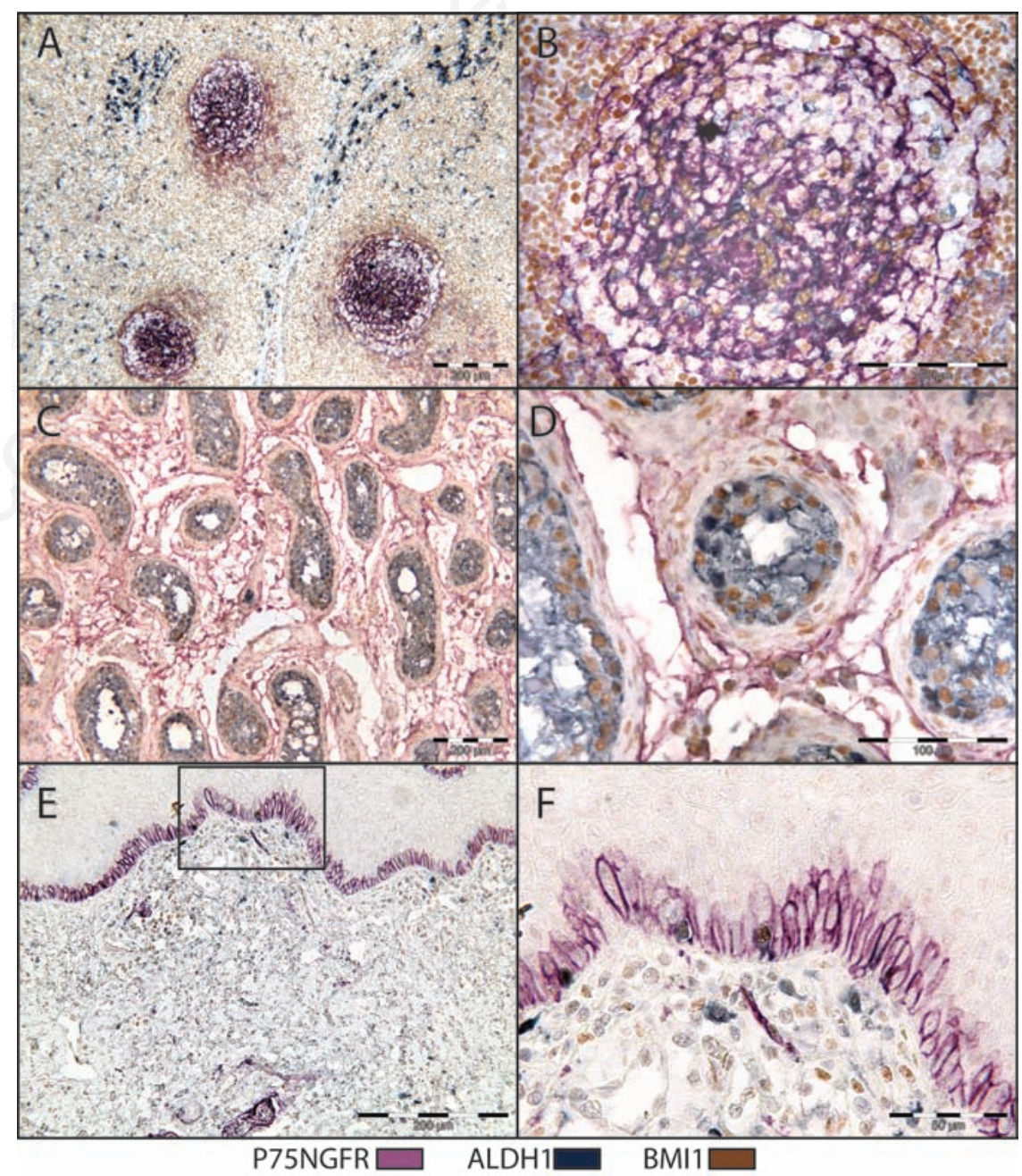

Figure 6. Triple IHC for P75NGFR (purple), BMI1 (brown) and ALDH1 (gray). Microwave heating was used for denaturation of preceding reaction complex. Cervical lymph node $(A, B)$, human testis $(C, D)$ and normal human oral mucosa $(E, F)$. 
cytoplasmic reaction has been visualized with other chromogens.

\section{Color deconvolution}

Sections that had undergone triple IHC with denaturation steps showed no visible crossreactivity under the light microscope, and were therefore included in the color deconvolution process. The input parameters obtained from single stained structures were found to be adequate for successful deconvolution of brown and purple, while further adjustments of the input parameters were necessary for deconvolution of the gray color, due to similar average blue optical density of the gray and purple. Separation of the three colors was eventually achieved, and the markup images were found to be consistent with the original digital slide (Figure 9).

\section{Discussion}

In many circumstances, multiple IHC can be a powerful tool in both diagnostics and research. However, the time and effort generally required for optimization of the procedure has influenced its attractiveness. Generally, reoptimizing the single IHC protocol for individual primary antibody is often mandatory to incorporate it in a multiple staining protocol. Firstly, the epitope retrieval method required for one antibody can have adverse effects on other epitopes. Finding a way around that frequently requires further search for the right antibody dilution and unspecific binding control method. Secondly, the choice of colors that can give good contrast has to be selected carefully, which might also require further adjustments for single staining protocol. These are basic processes that have to be carried out in every multiple IHC experiment. However, employing antibodies lacking the criteria necessary to selectively detect them by the secondary immunoreagents is more challenging. Even though ready-to-use kits for double IHC are commercially available, expanding the procedure into a triple or a quadruple staining requires procedures beyond the instructions that come with the kit. Although the sheltering effect of DAB has been employed in several multiple immunohistochemistry reports, ${ }^{5,23}$ cross reactivity below the visible limit of the human eye has not been investigated by computerized color unmixing methods, like Nuance software ${ }^{6}$ (Cambridge Research Instr., MA, USA) and Color co-localization algorithm (Aperio). Van der Loos reported that the sheltering effect is inversely related to primary antibody concentration. ${ }^{6}$ To make use of this effect, high concentrations of primary antibodies have to be avoided to lower the availability of probable sites of cross reactivity. In our

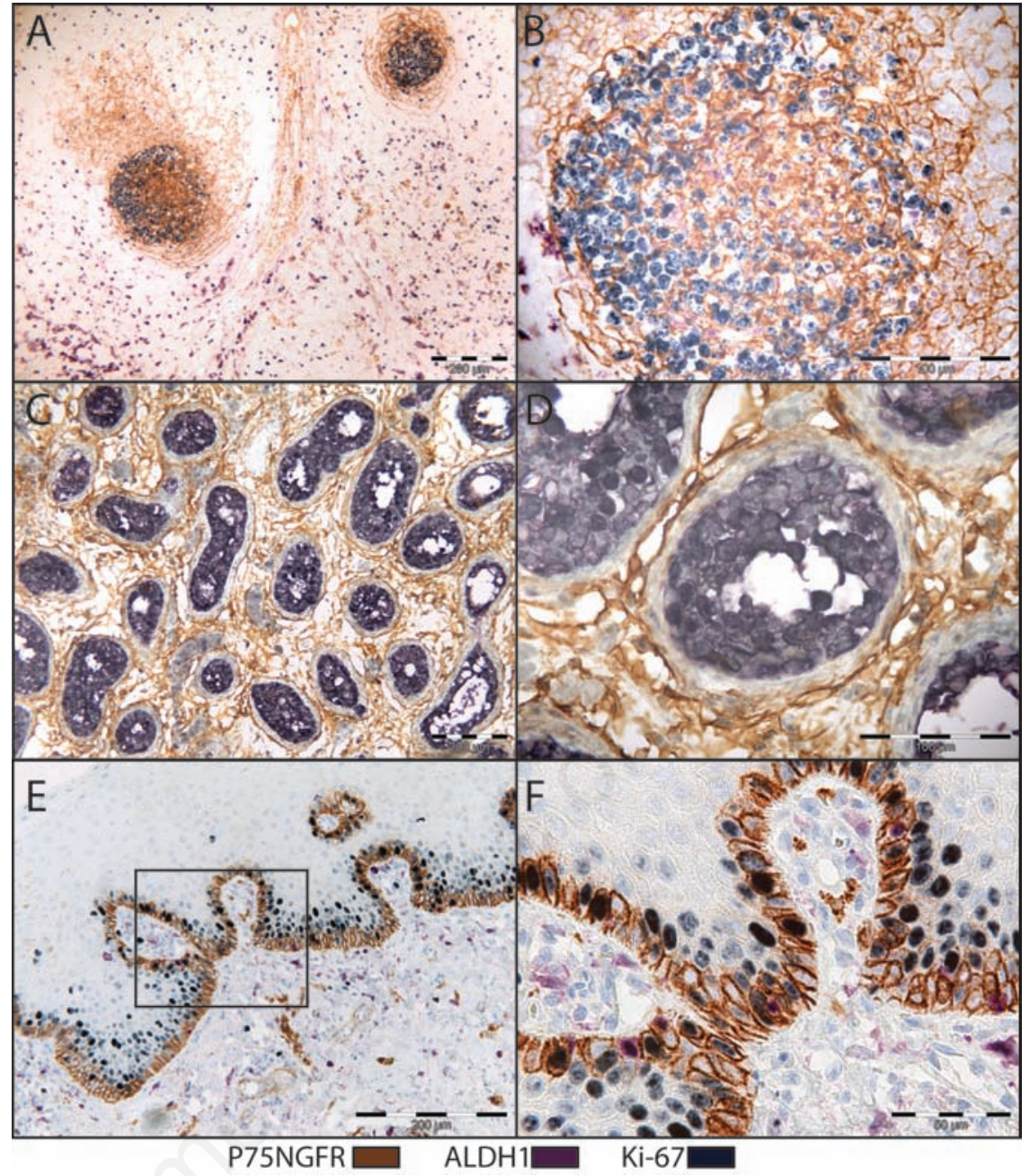

Figure 7. Triple IHC for P75NGFR (brown), Ki-67 (gray), ALDH1 (purple). Microwave heating was used for denaturation of preceding reaction complex. Cervical lymph node $(A, B)$, human testis $(C, D)$ and normal human oral mucosa $(E, F)$.

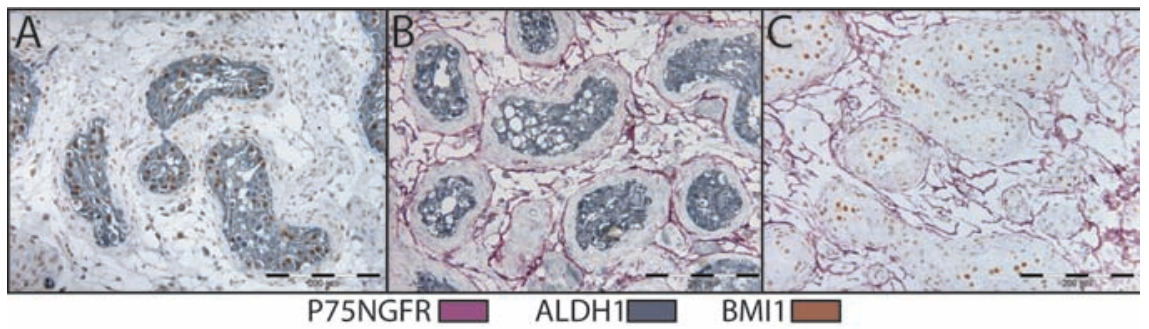

Figure 8. Triple IHC for P75NGFR (purple), BMI1 (brown) and ALDH1 (gray), in human testis. Microwave heating was used for denaturation of preceding reaction complex. Negative Control mouse IgG1 was used instead of: anti-P75NGFR (A), anti-BMI1 (B), and anti-ALDH1 (C) antibodies. 
experiment, reaction products of anti-BMI1 and anti-Ki67 antibodies were detected exclusively in the nucleus. Visualizing these reactions with DAB resulted in no immunoreactivity of subsequent reactions in the cytoplasm, when 1:500 dilution was used. Inversely, higher dilution (1:1500) of anti-BMIl antibody allowed subsequent cytoplasmic immunoreactivity. However, cross-reactivity with antiP75NGFR antibody targeting a cell surface receptor was evident by a mixed color in the nuclei, unless a denaturation step was performed. It seems that heavy deposition of DAB somehow affects the epitopes in close proximity. When anti-Ki67 antibody was investigated the same way, 1:1500 dilution was not always sufficient to maintain cytoplasmic antigenicity for subsequent reaction. Instead, anti-ki67 antibody reaction was carried out as the third one. The use of fragmented antibodies for saturation of preceding immunoreaction components requires prior knowledge of the antibody fragment needed according to the specificity of the secondary immunoreagents. Nowadays, the EnVision+ ${ }^{\circledR}$ kit from Dako is a commonly used detection method since the polymer technology employed by it, aids in intensifying the signal with no need for additional signal amplifier. Information about specificity of the polymer conjugated goat anti-mouse antibod-
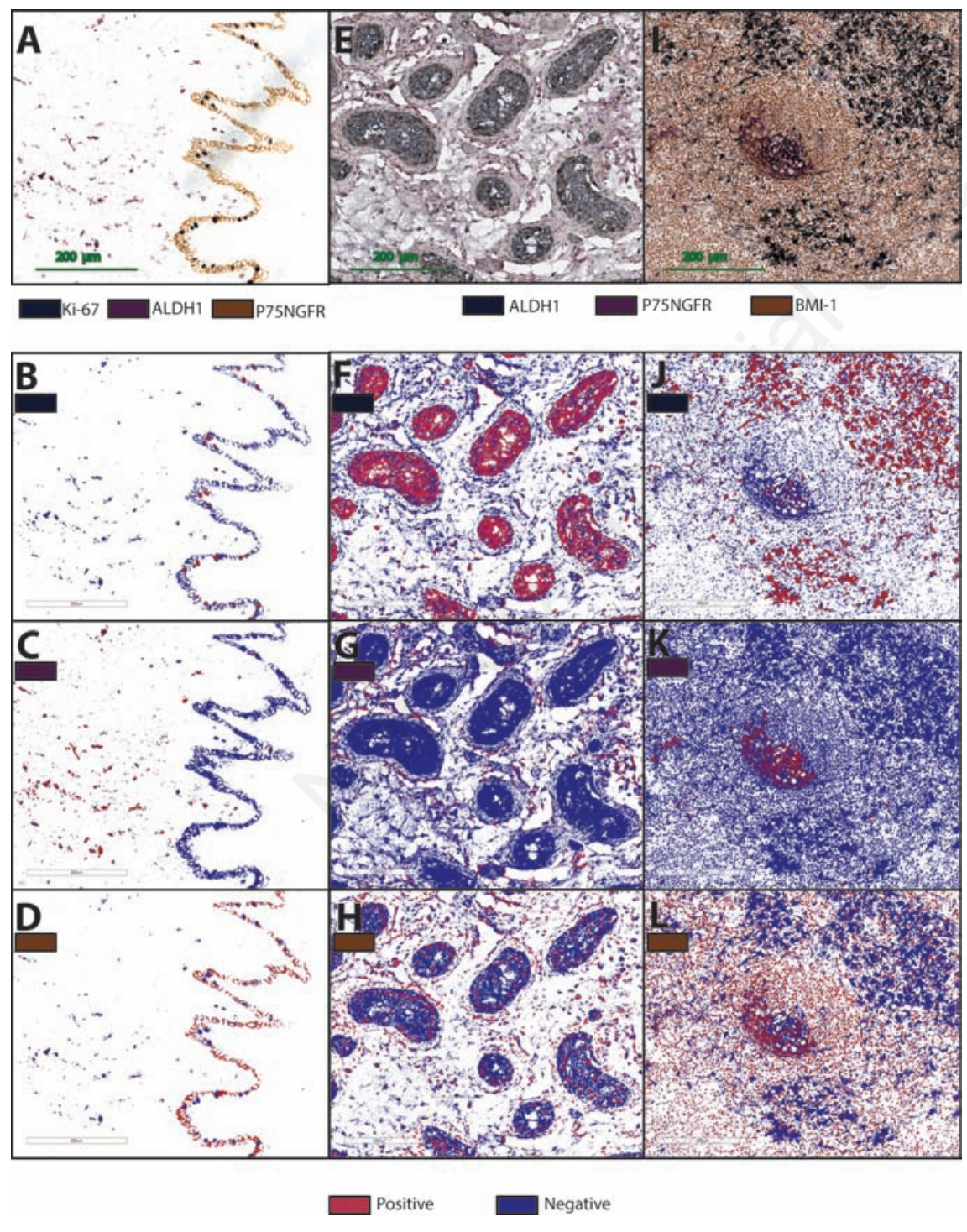

Figure 9. Reactions products of P75NGFR (brown), ALDH1 (purple) and Ki-67 (gray) in normal human oral mucosa (A), and P75NGFR (purple), ALDH1 (gray) and BMI1 (brown) in human testis (E) and cervical lymph node (I). Respective markup images for gray $(\mathrm{B}, \mathrm{F}, \mathrm{J})$, purple $(\mathrm{C}, \mathrm{G}, \mathrm{K})$ and brown $(\mathrm{D}, \mathrm{H}, \mathrm{L})$ as obtained from Aperio Color Deconvolution Algorithm. All positive structures are displayed in red. ies for $F(a b)$ or $F(c)$ fragment of mouse IgG is not available. Therefore, we used a whole nonbinding mouse IgG1 molecule for saturation of the preceding reaction complex. In order to prevent cross-binding with subsequent secondary immunoreagents, Doublestain Block from Dako was used afterwards. Nonetheless, cross-reactions evident by false double stained structures were detected when the streptavidin-biotin method was involved (Figure 3). The function of Doublestain Block is not revealed by the manufacturer, and its use is only recommended as part of a whole kit employing LP-HRP for the first reaction and LP-AP for a subsequent one. In this report, we investigated the ability of this reagent to block cross reactivity between successive reactions detected by other secondary immunoreagents. In accordance with the company recommendations, we found this reagent ineffective when the biotin-streptavidin method was used to detect one of the reactions. The scientific explanation of this is difficult to achieve since the mechanism of the reagent is not publicly available in the first place. When microwave oven is used for denaturation of antibodies, it is critical here to balance the required time to prevent cross-reactivity, but not to destroy tissue morphology, epitopes of subsequent reactions or to remove chromogens that have already been deposited from preceding reactions. The protocol reported in the 1990 s by Lan et al. ${ }^{12}$ destroyed some of the antigens in formalin fixed paraffin embedded samples, although this could be due to a difference in the microwave technologies from that time and now. Recently, a publication reported the use of microwave for double staining. ${ }^{13}$ In that study, targeted antigens were expressed by different types of cells. Applying the same method for triple staining of antigens in close proximity, was not sufficient to eliminate falsely double stained structures. However, additional use of peroxidase block enabled conducting the triple staining without spurious color. This indicated that the mixed color was due to a remaining enzyme activity. The effect of heating in the morphology of our tissues was considered acceptable, and the disadvantage was the difficulty in counterstaining them as compared to single stained sections. To get insight into the mechanism by which microwave oven prevents cross-reactivity, it is worth mentioning that studies on the effect of heat on immunoglobulin in breast milk showed that the $F(a b)$ region of $\operatorname{IgG}$, which includes the antigen binding site, undergoes denaturation at $61^{\circ} \mathrm{C}$, while denaturation of the $\mathrm{Fc}$ region takes place at $71^{\circ} \mathrm{C} .^{24}$ Secondly, maintaining the temperature at $77^{\circ} \mathrm{C}$ for 4.75 min was found to decreases the antigen binding activity of IgG by $90 \%{ }^{25}$ Thirdly, it was reported that stripping of tissue bound Ig's was only evident 
after several rounds of microwave oven heating. ${ }^{8}$ From the above, it seems that denaturation of the $F(a b)$ region is the first event happening during a microwave heating cycle, followed by denaturation of the Fc region, while the stripping of the Ig from its binding site may eventually occur if the microwave heating is prolonged. This is of special importance when performing IF staining, since stripping of Igs denotes loss of the fluorescent signal as well. In the current work, the temperature readings (Figure 5) showed that the temperature of the buffer exceeded the denaturation points of both $\mathrm{F}(\mathrm{ab})$ and $\mathrm{Fc}$ regions of $\mathrm{IgG}$, indicating that the cross-reactivity was accordingly inhibited.

The three reactions developed in this study, were often detected at different subcellular compartments of the same cell (Figure $6 \mathrm{~A}, \mathrm{C}$ ). Co-localization of ALDH1 with BMI1 or Ki-67 in the nucleus was neither visible under the regular light microscope, nor detectable by the color deconvolution process. Computerized color unmixing could be a powerful tool to analyze such color mixture at the same subcellular compartment. ${ }^{6}$ When the software required is not available, and such co-localization is important, many other useful color combinations can be employed. ${ }^{1,6}$ In our case, we have not investigated the applicability of color unmixing at sites of co-localization. Nevertheless, color deconvolution process enabled the separation of brown, purple and gray from different subcellular compartments into three individual images, usable for objective quantification and further analysis. The combination of brown (DAB) for a nuclear reaction and the gray (ImmPACT SG) for a cytoplasmic one, showed a sufficient contrast. Comparatively, the contrast of the gray color (ImmPACT SG) in the nucleus with the purple (ImmPACT VIP) for the cytoplasm, was found inferior in cases of low antigenicity of the nucleus. In conclusion, we suggest the use of microwave for denaturation of preceding reaction as a straight forward, practical, and affordable approach for simultaneous detection of multiple antigens in the same tissue section. This method also has the advantage of using the same secondary immunoreagent for detection of all reactions. Nevertheless, when applying this protocol to other experiments, certain points have to be considered carefully. Firstly, localization of antigens has to be investigated individually by single IHC, and expected sites of co-localization have to be taken into consideration. Secondly, all antibodies have to be optimized to work with the same epitope retrieval method. Thirdly, the order of antibodies, and color choice for each reaction has to be decided on their ability to withstand the microwave heating, as well as their effect on tissue antigenicity.

\section{References}

1. van der Loos CM. Immunoenzyme Multiple Staining Methods. UK: BIOS Scientific Publishers Limited; 1999.

2. Wallace EF, Wofsy L. Hapten-sandwich labeling. IV. Improved procedures and non-crossreacting hapten reagents for double-labeling cell surface antigens. J Immunol Methods 1979:25:283-9.

3. Negoescu A, Labat-Moleur F, Lorimier P, Lamarcq L, Guillermet C, Chambaz E, et al. F(ab) secondary antibodies: a general method for double immunolabeling with primary antisera from the same species. Efficiency control by chemiluminescence. J Histochem Cytochem 1994;42:433-7.

4. Renshaw $\mathrm{S}$, editor. Immunohistochemistry. Oxfordshire: Scion Publishing Ltd; 2007.

5. Sternberger LA, Joseph SA. The unlabeled antibody method. Contrasting color staining of paired pituitary hormones without antibody removal. J Histochem Cytochem 1979; 27:1424-9.

6. van der Loos CM. Multiple immunoenzyme staining: methods and visualizations for the observation with spectral imaging. $\mathrm{J}$ Histochem Cytochem 2008;56:313-28.

7. Lewis Carl SA, Gillete-Ferguson I, Ferguson DG. An indirect immunofluorescence procedure for staining the same cryosection with two mouse monoclonal primary antibodies. $\mathrm{J}$ Histochem Cytochem 1993;41:1273-8.

8. Tornehave D, Hougaard DM, Larsson L. Microwaving for double indirect immunofluorescence with primary antibodies from the same species and for staining of mouse tissues with mouse monoclonal antibodies. Histochem Cell Biol 2000;113:19-23.

9. Nakane PK. Simultaneous localization of multiple tissue antigens using the peroxidase-labeled antibody method: a study on pituitary glands of the rat. J Histochem Cytochem 1968;16:557-60.

10. Tramu G, Pillez A, Leonardelli J. An efficient method of antibody elution for the successive or simultaneous localization of two antigens by immunocytochemistry. J Histochem Cytochem 1978;26:322-4.

11. Pirici D, Mogoanta L, Kumar-Singh S, Pirici I, Margaritescu C, Simionescu C, et al. Antibody elution method for multiple immunohistochemistry on primary antibodies raised in the same species and of the same subtype. $\mathbf{J}$ Histochem Cytochem 2009;57: 567-75.

12. Lan HY, Mu W, Nikolic-Paterson DJ, Atkins RC. A novel, simple, reliable, and sensitive method for multiple immunoenzyme staining: use of microwave oven heating to block antibody crossreactivity and retrieve antigens. J Histochem Cytochem 1995;43:97-102. 13. Chan A, Matias MA, Farah CS. A novel and practical method using HRP-polymer conjugate and microwave treatment for visualization of 2 antigens raised from the same or different species in paraffin-embedded tissues. Appl Immunohistochem Mol Morphol 2011;19:376-83.

14. Labouyrie E, Parrens M, de Mascarel A, Bloch B, Merlio JP. Distribution of NGF receptors in normal and pathologic human lymphoid tissues. J Neuroimmunol 1997;77:161-73.

15. Raaphorst FM, van Kemenade FJ, Fieret E, Hamer KM, Satijn DP, Otte AP, et al. Cutting edge: polycomb gene expression patterns reflect distinct $B$ cell differentiation stages in human germinal centers. J Immunol 2000; 164:1-4.

16. Sanchez-Beato M, Sanchez E, GonzalezCarrero J, Morente M, Diez A, Sanchez-Verde L, et al. Variability in the expression of polycomb proteins in different normal and tumoral tissues. A pilot study using tissue microarrays. Mod Pathol 2006;19:684-94.

17. Vega JA, Garcia-Suarez 0, Hannestad J, PerezPerez M, Germana A. Neurotrophins and the immune system. J Anat 2003;203:1-19.

18. Lopez-Fernandez LA, del Mazo J. The cytosolic aldehyde dehydrogenase gene (Aldh1) is developmentally expressed in Leydig cells. FEBS Lett 1997;407:225-9.

19. Muller D, Davidoff MS, Bargheer O, Paust HJ, Pusch W, Koeva Y, et al. The expression of neurotrophins and their receptors in the prenatal and adult human testis: evidence for functions in Leydig cells. Histochem Cell Biol 2006;126:199-211.

20. Sugimoto R, Nabeshima Y, Yoshida S. Retinoic acid metabolism links the periodical differentiation of germ cells with the cycle of Sertoli cells in mouse seminiferous epithelium. Mech Dev 2012;128:610-24.

21. Nakamura T, Endo K, Kinoshita S. Identification of human oral keratinocyte stem/progenitor cells by neurotrophin receptor p75 and the role of neurotrophin/p75 signaling. Stem Cells 2007;25:628-38.

22. Uhlen M, Bjorling E, Agaton C, Szigyarto CA, Amini B, Andersen E, et al. A human protein atlas for normal and cancer tissues based on antibody proteomics. Mol Cell Proteomics 2005;4:1920-32.

23. Szyszko EA, Brokstad KA, Oijordsbakken G, Jonsson MV, Jonsson R, Skarstein K. Salivary glands of primary Sjogren's syndrome patients express factors vital for plasma cell survival. Arthritis Res Ther 2011;13:R2.

24. Vermeer AW, Norde W. The thermal stability of immunoglobulin: unfolding and aggregation of a multi-domain protein. Biophysical J 2000;78:394-404.

25. Dominguez E, Perez MD, Calvo M. Effect of heat treatment on the antigen-binding activity of anti-peroxidase immunoglobulins in bovine colostrum. J Dairy Sci 1997;80:3182-7. 